\title{
Socioeconomic Disparities in the Utilization of Mechanical Thrombectomy for Acute Ischemic Stroke in US Hospitals
}

\author{
W. Brinjikji, A.A. Rabinstein, J.S. McDonald, and H.J. Cloft
}

\begin{abstract}
BACKGROUND AND PURPOSE: Previous studies have demonstrated that socioeconomic disparities in the treatment of cerebrovascular diseases exist. We studied a large administrative data base to study disparities in the utilization of mechanical thrombectomy for acute ischemic stroke.
\end{abstract}

MATERIALS AND METHODS: With the utilization of the Perspective data base, we studied disparities in mechanical thrombectomy utilization between patient race and insurance status in 1) all patients presenting with acute ischemic stroke and 2) patients presenting with acute ischemic stroke at centers that performed mechanical thrombectomy. We examined utilization rates of mechanical thrombectomy by race/ethnicity (white, black, and Hispanic) and insurance status (Medicare, Medicaid, self-pay, and private). Multivariate logistic regression analysis adjusting for potential confounding variables was performed to study the association between race/insurance status and mechanical thrombectomy utilization.

RESULTS: The overall mechanical thrombectomy utilization rate was $0.15 \%(371 / 249,336)$; utilization rate at centers that performed mechanical thrombectomy was $1.0 \%$ (371/35,376). In the sample of all patients with acute ischemic stroke, multivariate logistic regression analysis demonstrated that uninsured patients had significantly lower odds of mechanical thrombectomy utilization compared with privately insured patients $(\mathrm{OR}=0.52,95 \% \mathrm{Cl}=0.25-0.95, P=.03)$, as did Medicare patients $(\mathrm{OR}=0.53,95 \% \mathrm{Cl}=0.41-0.70, P<.0001)$. Blacks had significantly lower odds of mechanical thrombectomy utilization compared with whites ( $\mathrm{OR}=0.35,95 \% \mathrm{Cl}=0.23-0.51, P<.0001)$. When considering only patients treated at centers performing mechanical thrombectomy, multivariate logistic regression analysis demonstrated that insurance was not associated with significant disparities in mechanical thrombectomy utilization; however, black patients had significantly lower odds of mechanical thrombectomy utilization compared with whites $(\mathrm{OR}=0.41,95 \% \mathrm{Cl}=0.27-0.60, P<.0001)$.

CONCLUSIONS: Significant socioeconomic disparities exist in the utilization of mechanical thrombectomy in the United States.

E ndovascular clot retrieval has become an increasingly utilized treatment for a select group of patients with acute ischemic stroke. ${ }^{1}$ Previous studies have demonstrated that significant socioeconomic disparities exist in the utilization of treatments such as tPA for acute ischemic stroke. ${ }^{2,3}$ With the use of the Perspective data base, we sought to determine if any socioeconomic disparities exist in the utilization of mechanical thrombectomy for the treatment of acute ischemic stroke.

\section{MATERIALS AND METHODS}

Patient Population

The Perspective data base is a voluntary, fee-supported collection of data developed by Premier, Inc (Charlotte, North Carolina), to

Received April 11, 2013; accepted after revision June 9

From the Departments of Radiology (W.B., J.S.M., H.J.C.), Neurology (A.A.R.), and Neurosurgery (H.J.C.), Mayo Clinic, Rochester, Minnesota.

Please address correspondence to Waleed Brinjikji, MD, Mayo Clinic, OL 1-115, 200 First St SW, Rochester, MN 55905; e-mail: brinjikji.waleed@mayo.edu

http://dx.doi.org/10.3174/ajnr.A3708 assess quality and resource utilization. As of 2011, the Perspective data base consisted of approximately $15 \%$ of hospitalizations nationwide and represented more than 600 US hospitals. Detailed information of a patient's hospitalization, including patient demographics, hospital information, diagnoses, procedures, discharge status, payer, and all billed items, are recorded.

Patients who presented with acute ischemic stroke (ICD9-CM diagnostic codes 433.x1 and 434.x1) from November 2005 through December 2011 were identified from the Perspective data base. Patients were only included if the stroke code was listed as the primary hospitalization diagnosis to avoid including patients with a history of stroke. Patients were stratified into 4 groups on the basis of insurance status: 1) uninsured, 2) Medicaid, 3) Medicare, and 4) private insurance, and 3 groups on the basis of race: 1) white, 2) black, and 3) Hispanic. Patients who received mechanical thrombectomy were identified by utilization of ICD-9 procedural code 39.74. We compared utilization rates of mechanical thrombectomy between insurance groups and racial groups. Other demographic variables and hospital characteristics 
Table 1: Demographic characteristics

\begin{tabular}{lcccc}
\hline & $\begin{array}{c}\text { All Patients } \\
\boldsymbol{n}(\%)\end{array}$ & $\begin{array}{c}\text { Thrombectomy } \\
\text { Patients } \boldsymbol{n}(\%)\end{array}$ & $\begin{array}{c}\text { Non-Thrombectomy } \\
\text { Patients } \boldsymbol{n}(\%)\end{array}$ & $\boldsymbol{P}$ \\
\hline Total No. of patients & 249,336 & $371(0.2)$ & $248,965(99.8)$ & - \\
Mean age, y (SD) & $71.6(14.0)$ & $66.6(16.3)$ & $71.6(14.0)$ & $<.0001$ \\
Sex & & & & .29 \\
$\quad$ Male & $114,199(45.8)$ & $185(49.9)$ & $114,014(45.8)$ & \\
$\quad$ Female & $135,137(54.2)$ & $186(50.1)$ & $134,951(54.2)$ & \\
Race & & & & \\
$\quad$ White & $159,673(76.5)$ & $270(86.5)$ & $159,403(76.5)$ & $<.0001$ \\
$\quad$ Black & $38,342(18.4)$ & $27(8.7)$ & $38,315(18.4)$ & \\
$\quad$ Hispanic & $10,738(5.1)$ & $15(4.8)$ & $10,723(5.1)$ & \\
Insurance status & & & & \\
$\quad$ Private & $38,741(15.5)$ & $95(25.6)$ & $38,646(15.5)$ & $<.0001$ \\
$\quad$ Medicare & $181,995(73.0)$ & $224(60.4)$ & $181,771(73.0)$ & \\
$\quad$ Medicaid & $16,540(6.6)$ & $39(10.5)$ & $16,501(6.6)$ & \\
$\quad$ Uninsured & $12,060(4.8)$ & $13(3.5)$ & $12,047(4.8)$ & \\
Hospital location & & & & \\
$\quad$ Urban & $222,497(89.2)$ & $361(97.3)$ & $222,136(89.2)$ & $<.0001$ \\
$\quad$ Rural & $26,839(10.8)$ & $10(2.7)$ & $26,829(10.8)$ & \\
Hospital teaching status & & & & \\
$\quad$ Teaching & $96,739(38.8)$ & $210(56.6)$ & $96,529(38.8)$ & $<.0001$ \\
$\quad$ Non-teaching & $152,597(61.2)$ & $161(43.4)$ & $152,436(61.2)$ & \\
\hline
\end{tabular}

208,753 patients. Of these, 159,673 patients $(76.5 \%)$ were white, 38,342 patients $(18.4 \%)$ were black, and 10,738 patients $(5.1 \%)$ were Hispanic. Mechanical thrombectomy utilization rate was $0.15 \%(371 / 249,336)$ in this sample. A total of 35,747 patients were treated at centers that performed mechanical thrombectomy. The utilization rate of mechanical thrombectomy at these centers was $1.0 \%(371 / 35,376)$. Demographic characteristics of the patients in this sample are summarized in Table 1.

\section{Insurance Status and Utilization}

When considering patients treated at all centers, mechanical thrombectomy utilization rates among private insurance patients was $0.24 \%(95 / 38,741)$. Mechanical thrombectomy utilization rate among Medicare patients was $0.12 \%$ (224/181,995), significantly lower than included in this study were sex, age, hospital location, and hospital teaching status.

Separate analyses were performed to determine disparities in the utilization rate of mechanical thrombectomy among 1) all patients with a primary diagnosis of acute ischemic stroke and 2) patients with acute ischemic stroke treated at centers that performed mechanical thrombectomy in a given year.

\section{Statistical Analysis}

$\chi^{2}$ tests were used to compare utilization rates of mechanical thrombectomy between insurance groups and race groups. For comparisons on the basis of insurance groups, the private insurance group was the reference group. For comparisons on the basis of race, white race was the reference. A multivariate logistic regression analysis was performed to study the association between insurance status and mechanical thrombectomy adjusting for patient age, race, sex, and hospital location and teaching status. A multivariate logistic regression analysis was performed to study the association between race and mechanical thrombectomy utilization, adjusting for patient age, insurance status, sex, hospital location, and teaching status. Multivariate logistic regression analyses were performed for the 2 separate samples 1 ) all patients with a primary diagnosis of acute ischemic stroke and 2) all patients with a primary diagnosis of acute ischemic stroke treated at centers offering thrombectomy. Multivariate outcomes are presented as odds ratios and $95 \%$ CIs. All statistical analysis was performed by use of the SAS-based software package JMP 9.0 (www.jmp.com).

\section{RESULTS}

\section{Patient Population}

A total of 249,336 patients were included in this study; 38,741 patients (15.5\%) had private insurance, 181,995 patients $(73.0 \%)$ had Medicare, 16,540 patients (6.6\%) had Medicaid, and 12,060 patients $(4.8 \%)$ were uninsured. Race data were available for that of private insurance patients $(P<.0001)$. Medicaid patients had a similar utilization rate of mechanical thrombectomy compared with private insurance patients $(39 / 16,540,0.24 \%, P=.92)$. Uninsured patients had significantly lower utilization rates of mechanical thrombectomy than private insurance patients (13/ 12,060, 0.11\%, $P=.005)$.

When we considered only patients treated at centers offering mechanical thrombectomy, mechanical thrombectomy utilization rates among private insurance patients was $1.4 \%$ (95/6947). Mechanical thrombectomy utilization rates among Medicare patients was $0.9 \%(224 / 24,756)$, significantly lower than that of private insurance patients $(P=.0008)$. Medicaid patients had a similar utilization rate of mechanical thrombectomy compared with private insurance patients (39/2545, $1.5 \%, P=.92)$. Uninsured patients had similar rates of mechanical thrombectomy compared with private insurance patients $(13 / 1499,0.9 \%, P=.13)$. These data are summarized in Table 2 .

\section{Race and Utilization}

When considering patients treated at all centers, mechanical thrombectomy utilization rate among white patients was $0.17 \%$ $(293 / 159,673)$. Black patients had a significantly lower rate of mechanical thrombectomy utilization compared with white patients (30/38,342, 0.07\%, $P<.0001)$. Hispanic patients had a similar utilization rate of mechanical thrombectomy compared with whites $(15 / 10,738,0.13 \%, P=.29)$.

When we considered only patients treated at centers offering mechanical thrombectomy, mechanical thrombectomy utilization rates among white patients was 1.2\% (293/24,988). Black patients had a significantly lower rate of mechanical thrombectomy utilization compared with white patients (30/4950, 0.6\%, $P=.0006)$. Hispanic patients had a similar utilization rate of mechanical thrombectomy compared with whites (15/1395, $1.1 \%, P=.84)$. These data are summarized in Table 2 . 
Table 2: Insurance status and race and mechanical thrombectomy utilization

\begin{tabular}{lccccc}
\hline & \multicolumn{2}{c}{ All Patients } & & \multicolumn{2}{c}{$\begin{array}{c}\text { Patients Treated at } \\
\text { Thrombectomy Centers }\end{array}$} \\
\cline { 2 - 3 } & $\boldsymbol{n}(\%)$ & $\boldsymbol{P}$ & & $\boldsymbol{n}(\%)$ & $\boldsymbol{P}$ \\
\hline Race & & & & \\
White & $293(0.17)$ & Reference & & $293.2)$ & Reference \\
Black & $30(0.07)$ & $<.0001$ & & $30(0.6)$ & .0006 \\
Hispanic & $15(0.13)$ & .29 & & $15(1.1)$ & .84 \\
Insurance & & & & Reference \\
Private & $95(0.24)$ & Reference & & $95(1.4)$ & .0008 \\
Medicare & $224(0.12)$ & $<.0001$ & & $224(0.9)$ & .61 \\
Medicaid & $39(0.24)$ & .92 & & $39(1.5)$ & .13 \\
None & $13(0.11)$ & .005 & $13(0.9)$ & \\
\hline
\end{tabular}

Table 3: Multivariate logistic regression analysis

\begin{tabular}{|c|c|c|c|c|}
\hline & \multicolumn{2}{|c|}{ All Patients } & \multicolumn{2}{|c|}{$\begin{array}{l}\text { Patients Treated at } \\
\text { Thrombectomy Centers }\end{array}$} \\
\hline & OR $(95 \% \mathrm{Cl})$ & $P$ & OR $(95 \% \mathrm{Cl})$ & $P$ \\
\hline \multicolumn{5}{|l|}{ Race $^{a}$} \\
\hline Black versus Hispanic & $0.42(0.23-0.81)$ & $<.0001$ & $0.50(0.26-0.97)$ & .04 \\
\hline Black versus white & $0.35(0.23-0.51)$ & $<.0001$ & $0.41(0.27-0.60)$ & $<.0001$ \\
\hline Hispanic versus white & $0.78(0.44-1.27)$ & .33 & $0.83(0.46-1.36)$ & .47 \\
\hline \multicolumn{5}{|l|}{ Insurance ${ }^{b}$} \\
\hline Medicare versus private & $0.53(0.41-0.70)$ & $<.0001$ & $1.12(0.80-1.57)$ & .52 \\
\hline Medicaid versus private & $1.09(0.70-1.65)$ & .71 & $1.19(0.76-1.82)$ & .43 \\
\hline None versus private & $0.52(0.25-0.95)$ & .03 & $0.63(0.31-1.18)$ & .16 \\
\hline Medicaid versus Medicare & $1.16(0.73-1.82)$ & .52 & $1.07(0.66-1.68)$ & .78 \\
\hline None versus Medicare & $0.56(0.27-1.05)$ & .07 & $0.57(0.27-1.08)$ & .08 \\
\hline None versus Medicaid & $0.48(0.22-0.95)$ & .03 & $0.94(0.60-1.51)$ & .78 \\
\hline
\end{tabular}

${ }^{a}$ Adjusted for insurance status, sex, age, hospital teaching status, and hospital location.

${ }^{\mathrm{b}}$ Adjusted for race, sex, age, hospital teaching status, and hospital location.

\section{Multivariate Analysis: All Patients}

After multivariate logistic regression analysis was performed, uninsured patients had a significantly lower odds of mechanical thrombectomy when compared with patients with private insurance $(\mathrm{OR}=0.52,95 \% \mathrm{CI}=0.25-0.95, P=.03)$. Medicare patients also had lower odds of mechanical thrombectomy utilization when compared with private insurance patients $(\mathrm{OR}=0.53$, 95\% CI $=0.41-0.70, P<.0001)$. Medicaid patients had a similar odds of mechanical thrombectomy utilization when compared with private insurance patients $(\mathrm{OR}=1.05,95 \% \mathrm{CI}=0.67-1.59$, $P=.83)$.

After multivariate logistic regression analysis examining race and outcomes was performed, black patients had a significantly lower odds of mechanical thrombectomy utilization compared with white patients $(\mathrm{OR}=0.35,95 \% \mathrm{CI}=0.23-0.51, P<.0001)$. Hispanic patients had a similar utilization rate of mechanical thrombectomy compared with whites $(\mathrm{OR}=0.78,95 \% \mathrm{CI}=$ $0.44-1.27, P=.33)$. These data are summarized in Table 3.

\section{Multivariate Analysis: Patients Treated at Thrombectomy Centers}

When examining patients treated at thrombectomy centers, there was no significant difference in thrombectomy utilization rates by insurance status. When examining odds of utilization by race, black patients had a significantly lower odds of mechanical thrombectomy utilization when compared with Hispanic patients $(\mathrm{OR}=0.50,95 \% \mathrm{CI}=0.26-0.97, P=.04)$. Blacks also had a significantly lower odds of receiving mechanical thrombectomy when compared with white patients $(\mathrm{OR}=$
$0.41,95 \% \mathrm{CI}=0.27-0.60, P<.0001)$. There was no significant difference in mechanical thrombectomy utilization when comparing white and Hispanic patients. These data are summarized in Table 3.

\section{DISCUSSION}

Our study demonstrated that significant race- and insurance-based disparities exist in the utilization of mechanical thrombectomy for treatment of acute ischemic stroke. On our adjusted analyses, uninsured and Medicare patients had significantly lower odds of mechanical thrombectomy utilization when compared with their privately insured counterparts. Similarly, black patients had significantly lower odds of mechanical thrombectomy utilization when compared with white and Hispanic patients. When we performed our adjusted analysis only examining thrombectomy utilization of patients treated at hospitals offering thrombectomy, some of these disparities remained present. Although insurance status was no longer associated with thrombectomy utilization, black patients were significantly less likely to receive mechanical thrombectomy than were their white and Hispanic counterparts. These findings suggest that disparities in mechanical thrombectomy utilization in the United States can be explained, in part, by lack of access to centers offering this treatment.

Studies examining racial and economic disparities in utilization of mechanical thrombectomy for acute ischemic stroke are scarce. One prior study of 602 patients found that blacks were significantly less likely to undergo endovascular interventions at stroke centers; however, when adjusted for the fact that African Americans had more delayed arrivals to the emergency department, they found no racial disparity. ${ }^{4}$ In general, socioeconomic status has been associated with significant disparities in the resource utilization for acute ischemic stroke. Patients of lower socioeconomic status have been shown to receive less emergent imaging, lower rates of hospital admission, and lower rates of poststroke care. ${ }^{3,5-7}$ Several prior studies have demonstrated significant socioeconomic disparities in the utilization of intravenous rtPA for the treatment of acute ischemic stroke. ${ }^{2,8-10}$ Nasr et $\mathrm{al}^{10}$ found that whites had significantly higher intravenous tPA treatment rates for acute ischemic stroke when compared with blacks and Hispanics in the United States. Johnston et $\mathrm{al}^{9}$ found that blacks were significantly less likely to receive intravenous tPA for acute ischemic stroke and demonstrated that contraindications to treatment did not account for the differences in tPA utilization rates. Prior studies in both the United States and Canada have demonstrated that neighborhood income is not associated with any significant differences in initial stroke care, but these 
studies did not evaluate differences in mechanical thrombectomy utilization rates. $^{11,12}$

There are many potential factors that could account for these disparities. Prior studies have demonstrated that disparities in the treatment of acute ischemic stroke in general could be related to delays in treatment, education level, or differences in stroke etiology. ${ }^{2,13-15}$ Minority patients are more likely to present with higher severity of stroke symptoms than whites and also present with more small-vessel lacunar strokes, thus making them less amenable to mechanical thrombectomy. ${ }^{16-19}$ Lower rates of mechanical thrombectomy among Medicare recipients could be related to a less aggressive therapeutic approach in older patients rather than to any economic considerations. ${ }^{20}$

\section{Limitations}

There are a number of limitations to our study. To maintain consistency with the Perspective data base, we used the same racial/ ethnic terms defined in the data base (white, black, Hispanic); however, we acknowledge that the broad racial designations provided present a potential limitation. Other weaknesses of this study include the fact that we are unable to determine important variables such as stroke severity at presentation, time to presentation, patient eligibility for mechanical thrombectomy, and the presence of large-vessel occlusions. Because of our inability to differentiate between various stroke subtypes, and, given the fact that stroke subtypes vary significantly by race, the observed racial disparities in our study must be interpreted cautiously. We cannot exclude that a racial difference in the likelihood of consenting for endovascular therapy could have played a contributing role. Coding errors are also a potential limitation of this study, as they are with any study of a large administrative data base. ${ }^{21,22}$

\section{CONCLUSIONS}

Our study demonstrated that significant socioeconomic disparities exist in the utilization of mechanical thrombectomy for the treatment of acute ischemic stroke. To ensure that all segments of the population have equal access to optimal stroke care, further studies are needed to study the underlying causes of these disparities.

Disclosures: Alejandro A. Rabinstein-UNRELATED: Royalties: Elsevier, Comments: For authorship rights; Other: Boston Scientific, Comments: Event adjudication board member for trial PREVAIL. Jennifer S. McDonald_UNRELATED: Grants/Grants Pending: GE Healthcare, ${ }^{*}$ Comments: Investigator-initiated research grant. Harry J. Cloft-UNRELATED: Grants/Grants Pending: Cordis Endovascular, ${ }^{*}$ Comments: Site $\mathrm{PI}$ at enrolling site for SAPPHIRE (Stenting and Angioplasty with Protection in Patients at HIgh Risk for Endarterectomy) registry ( ${ }^{\star}$ money paid to institution).

\section{REFERENCES}

1. Hassan AE, Chaudhry SA, Grigoryan M, et al. National trends in utilization and outcomes of endovascular treatment of acute ischemic stroke patients in the mechanical thrombectomy era. Stroke 2012;43:3012-17
2. Cruz-Flores S, Rabinstein A, Biller J, et al. Racial-ethnic disparities in stroke care: the American experience: a statement for healthcare professionals from the American Heart Association/American Stroke Association. Stroke 2011;42:2091-116

3. Addo J, Ayerbe L, Mohan KM, et al. Socioeconomic status and stroke: an updated review. Stroke 2012;43:1186-91

4. Bhattacharya P, Mada F, Salowich-Palm L, et al. Are racial disparities in stroke care still prevalent in certified stroke centers? J Stroke Cerebrovasc Dis 2013;22:383-88

5. Langagergaard V, Palnum KH, Mehnert F, et al. Socioeconomic differences in quality of care and clinical outcome after stroke: a nationwide population-based study. Stroke 2011;42:2896-902

6. Lazzarino AI, Palmer W, Bottle A, et al. Inequalities in stroke patients' management in English public hospitals: a survey on 200,000 patients. PLoS One 2011;6:e17219

7. Sandel ME, Wang $\mathrm{H}$, Terdiman J, et al. Disparities in stroke rehabilitation: results of a study in an integrated health system in northern California. PM\&R 2009;1:29-40

8. Fang MC, Cutler DM, Rosen AB. Trends in thrombolytic use for ischemic stroke in the United States. J Hosp Med 2010;5:406-09

9. Johnston SC, Fung LH, Gillum LA, et al. Utilization of intravenous tissue-type plasminogen activator for ischemic stroke at academic medical centers: the influence of ethnicity. Stroke 2001;32:1061-68

10. Nasr DM, Brinjikji W, Cloft HJ, et al. Racial and ethnic disparities in the use of intravenous recombinant tissue plasminogen activator and outcomes for acute ischemic stroke. J Stroke Cerebrovasc Dis 2013;22:154-60

11. Brown AF, Liang LJ, Vassar SD, et al. Neighborhood socioeconomic disadvantage and mortality after stroke. Neurology 2013;80:520-27

12. Kapral MK, Fang J, Chan C, et al. Neighborhood income and stroke care and outcomes. Neurology 2012;79:1200-07

13. Sallar AM, Williams PB, Omishakin AM, et al. Stroke prevention: awareness of risk factors for stroke among African American residents in the Mississippi delta region. J Natl Med Assoc 2010;102:84-94

14. Schwamm LH, Reeves MJ, Pan W, et al. Race/ethnicity, quality of care, and outcomes in ischemic stroke. Circulation 2010;121:1492-501

15. Anderson BE, Rafferty AP, Lyon-Callo S, et al. Knowledge of tissue plasminogen activator for acute stroke among Michigan adults. Stroke 2009;40:2564-67

16. Frey JL, Jahnke HK, Bulfinch EW. Differences in stroke between white, Hispanic, and Native American patients: the Barrow Neurological Institute stroke database. Stroke 1998;29:29-33

17. Jones MR, Horner RD, Edwards LJ, et al. Racial variation in initial stroke severity. Stroke 2000;31:563-67

18. Pathak EB, Sloan MA. Recent racial/ethnic disparities in stroke hospitalizations and outcomes for young adults in Florida, 2001-2006. Neuroepidemiology 2009;32:302-11

19. White H, Boden-Albala B, Wang C, et al. Ischemic stroke subtype incidence among whites, blacks, and Hispanics: the Northern Manhattan Study. Circulation 2005;111:1327-31

20. Saposnik G, Cote R, Phillips S, et al. Stroke outcome in those over 80: a multicenter cohort study across Canada. Stroke 2008;39:2310-17

21. Tirschwell DL, Longstreth WT Jr. Validating administrative data in stroke research. Stroke 2002;33:2465-70

22. Woodworth GF, Baird CJ, Garces-Ambrossi G, et al. Inaccuracy of the administrative database: comparative analysis of two databases for the diagnosis and treatment of intracranial aneurysms. Neurosurgery 2009;65:251-56 